\title{
Possible Tomography of the Sun's Magnetic Field with Solar Neutrinos
}

\author{
João Pulido \\ Centro de Física das Interacções Fundamentais \\ Instituto Superior Técnico \\ Av. Rovisco Pais, 1096 Lisboa Codex, Portugal
}

January 11, 2018

\begin{abstract}
The data from solar neutrino experiments together with standard solar model predictions are used in order to derive the possible profile of the magnetic field inside the Sun, assuming the existence of a sizeable neutrino magnetic moment and the resonant spin flavour mechanism. The procedure is based on the relationship between resonance location and the energy dependent neutrino suppression, so that a large neutrino suppression at a given energy is taken to be connected to a large magnetic field in a given region of the Sun. In this way it is found that the solar field must undergo a very sharp increase by a factor of at least 6 - 7 over a distance no longer than $7-10 \%$ of the solar radius, decreasing gradually towards the surface. The range in which this sharp increase occurs is likely to be the bottom of the convective zone. There are also indications in favour of the downward slope being stronger at the start and more moderate on approaching the solar surface. Typical ranges for the magnetic moment are from a few times $10^{-13} \mu_{B}$ to its laboratory upper bounds while the mass square difference between neutrino flavours is $\Delta^{2} m_{21} \simeq$ $(0.6-1.9) \times 10^{-8} \mathrm{eV}^{2}$.
\end{abstract}




\section{Introduction}

It is usually argued that the solar neutrinos which were first observed in 1968 [1] have not provided so far, as opposed to previous expectations, any particular knowledge of the physics inside the Sun. In fact the solar interior is opaque and detailed information about its structure has turned out possible to obtain, not via direct probes, but indirectly, via helioseismology [2].

Neutrinos however hold the potential of real probes of the solar interior, since they can travel across the Sun practically unperturbed. A neutrino produced near the centre of the Sun typically has a collision probability of the order of $10^{-6}-10^{-7}$ while travelling through the Sun, so the energy spread of a neutrino beam while traversing solar matter is left unaltered. If neutrinos are endowed with magnetic moment, an idea originally proposed by Cisneros [3] and later revived by Voloshin, Vysotsky and Okun 困, they can be converted via spin flip while travelling through the solar magnetic field into weakly sterile particles. Due to their neutrality the direction of propagation is undeflected.

One of the original motivations for this hypothesis was the apparent anticorrelation of neutrino flux in the Homestake experiment with sunspot activity [5. The data seemed to suggest that the more and the larger the sunspots, the fewer neutrinos were detected, indicating a more efficient spin flip conversion at times of more intense solar activity. Although this is an attractive hypothesis, several statistical analyses [6] - [9] fail to confirm this possibility and the data from all experiments [10] - [14] appear to be consistent with a constant rate 15.

It is now widely acknowledged that there exists a solar neutrino deficit which, in very general terms, may be stated as too few neutrinos being detected [10] - [14] as compared to theoretical predictions [16] - [21]. The possibility of a solution to this discrepancy within the minimal standard electroweak model appears highly unlikely. Hopes for solutions based on wrong interpretations of the experiments [10] - [14] or different input solar physics have successively been abandoned [22]. More and more the right solution seems to lie on non-standard neutrino properties such as oscillations (both MSW [23] or vacuum [24]), the resonant spin flavour precession mechanism (RSFP) [25] or flavour changing neutral current interactions [26]. The RSFP mechanism combines the original idea of Cisneros [3] with the interesting possibility of the conversion mechanism being a resonant one, like matter oscillations [23] and is totally consistent, as will be seen, with the absence of the above mentioned anticorrelation.

The present paper deals with RSFP type solutions to the solar neutrino problem. This mechanism requires a smaller magnetic field than the simple spin flip and one of its features is the fact that neutrinos of different energies have their resonances in different locations within the Sun, an essential point in the present analysis.

In the past few years of solar neutrino physics, it has become increasingly clear that the deficit is energy dependent [27] - [33], in the sense that weakly active neutrinos of different energies are suppressed differently. Since the resonance range is the zone where most if not all the conversion occurs and a stronger magnetic field leads to a greater suppression, an energy range where neutrinos are largely suppressed is a clear indication of a large magnetic field in a certain range of the Sun's interior. Conversely, a small suppression in a given energy range indicates a small magnetic field in the region where the corresponding resonance is located. This principle is the basic idea of the present work and in this way a 'tomography' of the solar magnetic field may be obtained. Hence the neutrino energy 
suppression spectrum is expected to be directly connected to the profile of the magnetic field along the Sun's radius, provided the neutrinos have a sizeable magnetic moment. It should be stressed that a sensible solution for the field profile is reasonably constrained, as it must account for a strong suppression for the intermediate energy neutrinos, a moderate one for the energetic ${ }^{8} B$ ones and no suppression for the $p p$ ones. This result together with the requirement that all neutrinos should have a resonance inside the Sun implies, as an essential feature, that the average magnetic field should undergo a discontinuity or at least a very fast variation in space somewhere in the interior of the Sun.

Recent progress in helioseismology seems to confirm the fact that the fluid character of the convective zone is interrupted near its bottom at approximately $0.71 R_{S}$. Below this depth a rigid body like structure appears. This is thus the likeliest region for the fast variation to arise. From the analysis of this paper one can predict that, starting from the solar surface where the field should not exceed a few $\mathrm{kG}$ in the sunspots, one expects an increase along the convective zone towards a maximum near its bottom. This increase appears to become more intense in the deeper layers of the convective zone. Proceeding inwards in the radial direction, a sudden drop by an order of magnitude or more over the following $7-10 \% R_{S}$ is expected. Below this range the field is either negligible or may rise very moderately. This expectation is consistent with a neutrino magnetic moment in the range from a few times $10^{-13} \mu_{B}$ up to its laboratory bound of $10.8 \times 10^{-10} \mu_{B}$ [34].

The paper is divided as follows: in section 2 a brief review of the experimental situation [10] - [14] is presented and its implications for the survival probabilities of low, intermediate and high energy neutrinos are derived using the predictions from several standard solar models [16] - 21]. In section 3 the neutrino propagation equation through solar matter is solved analytically and generalized to include the neutrino energy [35] and production zone distributions [21] and non-adiabatic effects in the form of the Landau Zener approximation [36]. The expression of the survival probability is next evaluated. In section 4 the necessary general profile which is required in order to satisfy experimental data and the results from solar models is established. It is argued that the high suppression found for the intermediate energy neutrinos and the absence of suppression for the low energy ones along with the fact that their energies are relatively close is an indication of the sudden field variation referred to above. An example of a toy magnetic field is obtained from approximate general conditions. In section 5 an outlook is presented. Majorana neutrinos with transition magnetic moments $\left(\nu_{e} \rightarrow \bar{\nu}_{\mu}\right)$ will be used throughout, the results for Dirac neutrinos being practically identical [37.

\section{Energy Dependent Solar Neutrino Suppression}

The data from the four solar neutrino experiments [10] - [14 are quoted in table I and the corresponding predictions from six solar models [16] - 21] including the contribution from each neutrino component are given in table II. All models include heavy element diffusion except for TCL [16] and TCCCD [17]. It is now generally acknowledged that a 'standard' solar model (SSM) should include diffusion, owing to the fact that such models give a remarkably good agreement with data from helioseismology [2].

The absence of the intermediate energy neutrinos, consisting principally of the ${ }^{7} \mathrm{Be}$ line at $E=0.86 \mathrm{MeV}$ and the $\mathrm{CNO}$ continuum, has been realized several years ago [29] from the comparison of the Homestake and Kamiokande data. It is considered by some as 
the 'true' solar neutrino problem in the sense that it is independent from normalization to any solar model, either standard or non-standard [38]. It appears as a natural consequence of the luminosity constraint 21], 32] $\left(L_{\odot}=1.367 \times 10^{-1} \mathrm{Wcm}^{-2}\right)$

$$
L_{\odot}=\sum_{k}\left(\frac{Q}{2}-<E_{\nu}>_{k}\right) f_{k} \quad\left(k=p p, p e p,{ }^{7} B e, C N O,{ }^{8} B\right)
$$

with $Q=26.73 \mathrm{MeV}$ (total energy released in each neutrino pair production) and the equations [32]

$$
\begin{aligned}
S_{G a} & =\sum_{i} \sigma_{G a, i} f_{i} \quad\left(i=p p, p e p,{ }^{7} B e, C N O,{ }^{8} B\right) \\
S_{C l} & =\sum_{j} \sigma_{C l, j} f_{j} \quad\left(i={ }^{7} B e, C N O,{ }^{8} B\right) \\
f_{p e p} & =0.0023 f_{p p}
\end{aligned}
$$

where $S_{G a}, S_{C l}$ denote the Gallium and Chlorine data.

One has in fact from this system of four equations, upon elimination of the $p p$ flux $f_{p p}$, using the average value $\bar{S}_{G a}=73.8 \pm 7.7 S N U$ and the nuclear cross sections [32] $\sigma_{G a, i}, \sigma_{C l, j}$, the following intermediate energy neutrino flux (in units $\mathrm{cm}^{-2} \mathrm{~s}^{-1}$ )

$$
\begin{aligned}
f_{B e} & =1.04 \times 10^{4} f_{B}-2.88 \times 10^{10} \\
f_{C N O} & =-8.46 \times 10^{3} f_{B}+2.22 \times 10^{10} .
\end{aligned}
$$

Inserting $f_{B}$ from SuperKamiokande, [14] the total flux from these neutrinos is negative:

$$
\begin{aligned}
f_{B e} & =-3.42 \times 10^{10} \mathrm{~cm}^{-2} \mathrm{~s}^{-1} \\
f_{C N O} & =1.56 \times 10^{10} \mathrm{~cm}^{-2} \mathrm{~s}^{-1} .
\end{aligned}
$$

Better fits were done by the authors of [32, [31] who obtained

$$
\begin{aligned}
& f_{B e+C N O} \leq 0.7 \times 10^{9} \mathrm{~cm}^{-2} s^{-1} \quad(3 \sigma) \\
& f_{B e+C N O}=(-2.5 \pm 1.1) \times 10^{9} \mathrm{~cm}^{-2} s^{-1}
\end{aligned}
$$

These authors used the former Kamiokande flux data which were higher than SuperKamiokande. From equations (5) and (6) it is seen that the total flux $f_{B e+C N O}$ decreases with decreasing $f_{B}$, so that the results $(9),(10)$ should be further aggravated in the nonphysical direction. Hence the probability that neutrinos are standard is no greater than 1\%, while, if the luminosity constraint is dropped, it may increase to 4\% [31]. So intermediate energy neutrinos appear in practice to be completely suppressed.

As far as high energy $\left({ }^{8} B\right)$ neutrinos are concerned and denoting by $R_{C l}$ the ratio data/SSM prediction one may write

$$
R_{C l}=R_{C l}^{I} P_{I}+R_{C l}^{H} P_{H}
$$

Here $R_{C l}^{I(H)}$ is the fraction of intermediate (high) energy neutrinos in the Chlorine experiment as theoretically predicted and $P_{I(H)}$ is the fraction of intermediate (high) energy $\nu_{e_{L}}$ produced in the Sun that are detected on Earth. Using $P_{I}=0$ (99\%CL) and the models listed in table I one gets for $P_{H}$ the range

$$
0.35<P_{H}<0.63
$$


with the smaller value corresponding to BP95 [21] and the larger to TCCCD [17].

The same argument may now be applied to the low energy $(p p)$ neutrinos. With obvious notation, one has for the Gallium result

$$
R_{G a}=R_{G a}^{L} P_{L}+R_{G a}^{I} P_{I}+R_{G a}^{H} P_{H}
$$

Again, taking $P_{I}=0$, one obtains for the two extreme cases

$$
\begin{array}{ll}
P_{L}=0.981 & (\mathrm{BP} 95)[21] \\
P_{L}=0.966 & (\text { TCCCD })[17
\end{array}
$$

which means that the low energy $(p p)$ neutrinos are practically unsuppressed. Thus any solution to the solar neutrino problem based on non-standard neutrino properties must be consistent with the following general conclusions:

1. No suppression for low energy $(p p)$ neutrinos.

2. Strong suppression for intermediate energy $\left({ }^{7} B e, \mathrm{CNO}\right)$ neutrinos.

3. Moderate suppression for high energy $\left({ }^{8} B\right)$ neutrinos.

The requirements $P_{L}>0.96, P_{I}<0.04,0.35<P_{H}<0.63$ will be used throughout the paper.

\section{Survival Amplitudes and Probabilities}

The starting point of this section is the Schrödinger-like evolution equation for Majorana neutrinos in the Sun [37]

$$
-i \frac{d}{d r}\left(\begin{array}{c}
\nu_{e_{L}} \\
\bar{\nu}_{\mu_{R}}
\end{array}\right)=H\left(\begin{array}{c}
\nu_{e_{L}} \\
\bar{\nu}_{\mu_{R}}
\end{array}\right)
$$

with the Hamiltonian $\mathrm{H}$ given by

$$
H=\left(\begin{array}{cc}
\left(\frac{G}{\sqrt{2}}\right) \frac{11}{6} N_{e} & \mu B \\
\mu B & \frac{\Delta^{2} m_{21}}{2 E}+\left(\frac{G}{\sqrt{2}}\right) \frac{1}{6} N_{e}
\end{array}\right) .
$$

Here $\Delta^{2} m_{21}$ is the mass square difference between mass eigenstates, $N_{e}$ is the electron density taken as exponentially decreasing with radius in the region of interest [35], $G N_{e}=$ $2.11 \times 10^{-11} \exp \left(-r / 0.09 R_{S}\right) e V, \mathrm{~B}$ is the solar magnetic field and the remaining notation is obvious. A vanishing vacuum mixing angle is assumed throughout and the mixing angle from matter and magnetic moment/field is [37

$$
s^{2}{ }_{\theta}=\frac{\mu^{2} B^{2}}{\mu^{2} B^{2}+\frac{1}{4}\left[\frac{5 G}{3 \sqrt{2}} N_{e}-\frac{\Delta^{2} m_{21}}{2 E}+\left(\left(\frac{5 G}{3 \sqrt{2}} N_{e}-\frac{\Delta^{2} m_{21}}{2 E}\right)^{2}+4 \mu^{2} B^{2}\right)^{1 / 2}\right]^{2}} .
$$

The neutrino propagation through solar matter is assumed to be adiabatic except for a short range [37 in the neighbourhood of a critical point - the resonance - where the mixing angle (18) is maximal so that the density verifies

$$
\frac{5 G}{3 \sqrt{2}} N_{e}=\frac{\Delta^{2} m_{21}}{2 E}
$$


Across the resonance the neutrinos may shift from one eigenvalue to another, this effect being parametrized by a jump amplitude. So a neutrino produced as a weak eigenstate $\nu_{e_{L}}$ at $r_{i}$ which is a superposition of two mass eigenstates $\nu_{1}, \nu_{2}$

$$
\left(\begin{array}{c}
\nu_{1}\left(r_{i}\right) \\
\nu_{2}\left(r_{i}\right)
\end{array}\right)=\left(\begin{array}{cc}
c_{\theta_{i}} & s_{\theta i} \\
-s_{\theta_{i}} & c_{\theta i}
\end{array}\right)\left(\begin{array}{c}
1 \\
0
\end{array}\right)
$$

will become at location $r_{1}$, after having passed through a resonance [39], the following combination

$$
\left(\begin{array}{c}
\nu_{1}\left(r_{1}\right) \\
\nu_{2}\left(r_{1}\right)
\end{array}\right)=\left(\begin{array}{ccc}
\lambda_{1} \exp i \int_{r_{i}}^{r_{1}} H_{D_{11}} d r & \lambda_{2} \exp i \int_{r_{i}}^{r_{1}} H_{D_{22}} d r \\
-\lambda_{2}^{*} \exp i \int_{r_{i}}^{r_{1}} H_{D_{11}} d r & \lambda_{1}^{*} \exp i \int_{r_{i}}^{r_{1}} H_{D_{22}} d r
\end{array}\right)\left(\begin{array}{c}
\nu_{1}\left(r_{i}\right) \\
\nu_{2}\left(r_{i}\right)
\end{array}\right) .
$$

Here $\lambda_{1}$ denotes the amplitude for adiabatic propagation, $\lambda_{2}$ is the jump amplitude across the resonance and $H_{D_{11}}, H_{D_{22}}$ are the 11 and 22 elements of the diagonalized Hamiltonian. Denoting by $\theta_{1}$ the diagonalizing angle at $r_{1}$, it is straightforward to derive the survival amplitude, that is, the amplitude that a neutrino which is produced as $\nu_{e_{L}}$ remains as $\nu_{e_{L}}$

$$
\begin{aligned}
A m p\left(\nu_{e_{L}}\left(r_{i}\right) \rightarrow \nu_{e_{L}}\left(r_{1}\right)\right) & =c_{\theta_{1}} c_{\theta_{i}} \lambda_{1} \exp i \int_{r_{i}}^{r_{1}} H_{D_{11}} d r-c_{\theta_{1}} s_{\theta_{i}} \lambda_{2} \exp i \int_{r_{i}}^{r_{1}} H_{D_{22}} d r \\
& +s_{\theta_{1}} c_{\theta_{i}} \lambda_{2}^{*} \exp i \int_{r_{i}}^{r_{1}} H_{D_{11}} d r+s_{\theta_{1}} s_{\theta_{i}} \lambda_{1}^{*} \exp i \int_{r_{i}}^{r_{1}} H_{D_{22}} d r .
\end{aligned}
$$

For the conversion amplitude one just performs in (22) the replacements $c_{\theta_{1}} \rightarrow s_{\theta_{1}},-s_{\theta_{1}} \rightarrow$ $c_{\theta_{1}}$. In a simplified calculation where all neutrinos are considered to be emmited at the same point and with the same energy, the survival probability is simply the square modulus of eq. (22) where all fast oscillating terms are set equal to zero. This is the well known Parke's formula 39

$$
P_{\text {surv }}=\frac{1}{2}+\left(\frac{1}{2}-P_{C}\right) c_{2 \theta_{i}} c_{2 \theta_{1}}
$$

where the quantity $P_{C}$ is the jump probability, $P_{C}=\left|\lambda_{2}\right|^{2}$, which will be taken in the Landau Zener approximation [36, 40, ษ.

$$
P_{C}=\exp \left(-\pi \frac{2 \mu^{2} B^{2}}{\frac{\Delta^{2} m_{21}}{2 E}} 0.09 R_{S}\right)
$$

The coefficient of this exponent is the adiabaticity parameter,

$$
\gamma_{C}=\frac{2 \mu^{2} B^{2}}{\frac{\Delta^{2} m_{21}}{2 E}} 0.09 R_{S}
$$

A more accurate calculation must include the production and energy range of the neutrinos. Denoting by $\chi(E)$ and $\phi\left(r_{i}\right)$ the production amplitudes per unit energy and unit length respectively, the survival amplitude at the edge of the Sun $\left(r_{1}=R_{S}\right)$ becomes the sum of the following four terms

\footnotetext{
${ }^{1}$ It was previously shown that the Landau Zener approximation which assumes a linearly decreasing density in the vicinity of the critical point works rather well in the Sun. See [40]
} 


$$
\begin{aligned}
\nu_{e} & =\int_{E_{m}}^{E_{M}} \chi(E) c_{\theta_{1}} \int_{a}^{b} \lambda_{1} \phi\left(r_{i}\right) c_{\theta_{i}} \exp i \int_{r_{i}}^{R_{S}} H_{D_{11}} d r d r_{i} d E \\
& -\int_{E_{m}}^{E_{M}} \chi(E) c_{\theta_{1}} \int_{a}^{b} \lambda_{2} \phi\left(r_{i}\right) s_{\theta_{i}} \exp i \int_{r_{i}}^{R_{S}} H_{D_{22}} d r d r_{i} d E \\
& +\int_{E_{m}}^{E_{M}} \chi(E) s_{\theta_{1}} \int_{a}^{b} \lambda_{2}^{*} \phi\left(r_{i}\right) c_{\theta_{i}} \exp i \int_{r_{i}}^{R_{S}} H_{D_{11}} d r d r_{i} d E \\
& +\int_{E_{m}}^{E_{M}} \chi(E) s_{\theta_{1}} \int_{a}^{b} \lambda_{1}^{*} \phi\left(r_{i}\right) s_{\theta_{i}} \exp i \int_{r_{i}}^{R_{S}} H_{D_{22}} d r d r_{i} d E .
\end{aligned}
$$

while the conversion amplitude

$$
\begin{gathered}
\bar{\nu}_{\mu}=\int_{E_{m}}^{E_{M}} \chi(E) s_{\theta_{1}} \int_{a}^{b} \lambda_{1} \phi\left(r_{i}\right) c_{\theta_{i}} \exp i \int_{r_{i}}^{R_{S}} H_{D_{11}} d r d r_{i} d E \\
-\int_{E_{m}}^{E_{M}} \chi(E) s_{\theta_{1}} \int_{a}^{b} \lambda_{2} \phi\left(r_{i}\right) s_{\theta_{i}} \exp i \int_{r_{i}}^{R_{S}} H_{D_{22}} d r d r_{i} d E \\
-\int_{E_{m}}^{E_{M}} \chi(E) c_{\theta_{1}} \int_{a}^{b} \lambda_{2}^{*} \phi\left(r_{i}\right) c_{\theta_{i}} \exp i \int_{r_{i}}^{R_{S}} H_{D_{11}} d r d r_{i} d E \\
-\int_{E_{m}}^{E_{M}} \chi(E) c_{\theta_{1}} \int_{a}^{b} \lambda_{1}^{*} \phi\left(r_{i}\right) s_{\theta_{i}} \exp i \int_{r_{i}}^{R_{S}} H_{D_{22}} d r d r_{i} d E .
\end{gathered}
$$

In these expressions the first two integrals in each term extend over the energy and production range for each neutrino component $\left(p p,{ }^{7} \mathrm{Be},{ }^{8} \mathrm{~B}, \ldots\right)$ as given in refs. [35], [21] respectively (model with $\mathrm{He}$ and metal diffusion).

Turning now to the probabilities, it should first be observed that nuclei produce neutrinos in a disordered manner both in space-time and in energy. Each neutrino therefore acquires a random phase endowing the terms (26) - (29) and (30) - (33) which therefore add incoherently, as opposed to (22). The random neutrino emission thus makes the amplitudes completely incoherent, so one adds probabilities. The generalization of (23) including energy and production range distribution is thus

$$
P_{\text {surv }}=\int_{E_{m}}^{E_{M}} g(E) \int_{a}^{b} f\left(r_{i}\right)\left[\frac{1}{2}+\left(\frac{1}{2}-P_{C}\right)\right] c_{2_{\theta_{1}}} c_{2_{\theta_{i}}} d r_{i} d E .
$$

For the conversion probability one just performs in $(34)$ the replacement $(+) \rightarrow(-)$. The functions $g(E), f\left(r_{i}\right)$, representing respectively the probability density of neutrino production per unit energy and per unit length, will be taken from the numerical tables in refs. [35], [21]. An example of a survival probability showing the characteristic 'bath tub' shape is shown in fig. 1.

Equations (34), (24), (25) constitute the basis of the following analysis in section 4. 


\section{Magnetic Field Profiles}

\subsection{General Features}

Apart from the knowledge that neutrinos may provide us if they have a sizeable magnetic moment, very little is known about the solar magnetic field. From the observation of the interstellar field, a lower bound may be fixed at $10 \mu G$, whereas an upper bound of $(2-5) \times 10^{3} G$ near the surface may be considered as associated to the field in sunspots. There is however no indication as to the extension of these structures and its associated field in depth significantly below the surface layers.

Several 'plausible' profiles have been proposed and investigated in the litterature [41] [44 but these analyses which are now several years old did not take into account an energy dependent solar neutrino suppression. It should first be emphasized that the resonance condition (19) fixes the location of the critical density for each neutrino according to its own energy [37]:

$$
x_{\text {res }}=0.09 \log \frac{\frac{5}{3 \sqrt{2}} 2.11 \times 10^{-11} \mathrm{eV}}{\frac{\Delta^{2} m_{21}}{2 E}}
$$

where $x$ is the fraction of the solar radius $x=r / R_{S}$.

The range around the critical density where the Hamiltonian eigenvalue splitting,

$$
\alpha_{1}-\alpha_{2}=\sqrt{\left(\frac{5 G}{3 \sqrt{2}} N_{e}-\frac{\Delta^{2} m_{21}}{2 E}\right)^{2}+4 \mu^{2} B^{2}}
$$

is dominated by the term $4 \mu^{2} B^{2}$ is where most if not all the conversion (i. e. neutrino suppression) occurs.

An efficient conversion means that the jump probability (24) across the resonance from one Hamiltonian eigenvalue to the other is small, so each neutrino remains in its own eigenvalue. The process is therefore adiabatic and $\gamma_{C}$ (25) is large. Most of the neutrinos will gradually change their flavour and the space rate of this change is maximal at the vicinity of the critical point. Conversely, a sizeable jump probability means that a significant fraction of neutrinos will change from one Hamiltonian eigenvalue to the other, thereby staying in the same weak eigenstate and the process is non-adiabatic. In all resonant processes the resonance is of course located between the production zone and the surface of the Sun, so the mixing angle (18) at the production point is close to zero and at the edge of the Sun is close to $\pi / 2$ [37]. In the rough approximation (23) with no energy range nor production zone distribution, this means $c_{2 \theta_{i}} \simeq 1$ and $c_{2 \theta_{1}} \simeq-1$ so that

$$
P_{\text {surv }} \simeq P_{C} .
$$

A large jump probability opposes conversion, thus favouring survival. In such case most neutrinos, while travelling across the resonance, will leave to the other Hamiltonian eigenvalue which corresponds, past the resonance, mainly to the initial weak eigenstate. So neutrino survival is related to strongly adiabatic processes and conversion to the lack of adiabaticity. Recalling the analysis of section 2, these arguments show that $p p$ neutrinos, for which $P_{L} \geq 0.96$, must correspond to highly non-adiabatic resonances,

$$
\gamma_{C} \leq 0.01
$$


while intermediate energy neutrinos which are highly suppressed $\left(P_{I} \leq 0.04\right)$ must correspond to

$$
\gamma_{C} \geq 1 \text {. }
$$

Requiring condition (38) to hold for all $p p$ neutrinos whose energies lie in the interval

$$
0.236 \mathrm{MeV} \leq E_{p p} \leq 0.420 \mathrm{MeV}
$$

and taking intermediate energy neutrinos satisfying (39) to consist totally of the ${ }^{7} B e$ line at

$$
E_{7_{B e}}=0.86 \mathrm{MeV}
$$

one has respectively from (38), (39) using (25)

$$
\begin{array}{r}
0.09 R_{S} 2 \mu^{2} B^{2}\left(x_{7_{B e}}\right) \geq \frac{\Delta^{2} m_{21}}{2 E_{7_{B e}}} \\
0.09 R_{S} 2 \mu^{2} B^{2}\left(x_{p p}\right) \leq 0.01 \frac{\Delta^{2} m_{21}}{2 E_{p p}} .
\end{array}
$$

The solar magnetic field should satisfy these two conditions at the corresponding critical densities. Using the values for $E_{7 B e}=0.86 \mathrm{MeV}$ and $E_{p p_{\max }}=0.42 \mathrm{MeV}$, it is seen from (35) that the spacing between these critical densities is

$$
\Delta x_{7_{B e, p p}}=(0.065-0.116)
$$

implying a sharp rise of the magnetic field by at least a factor of (6 - 7) over a relatively short radial distance. Therefore it is seen from (42), (43) that the field is both bound from below and from above at points whose mutual distance is $\Delta x_{{ }^{7} B e, p p}$ :

$$
\begin{array}{r}
B\left(x_{{ }^{7} B e}\right) \geq \frac{5.22 \times 10^{-3}}{f} \sqrt{\Delta^{2} m_{21}} \\
B\left(x_{p p}\right) \leq \frac{0.483}{f} \sqrt{\frac{\Delta^{2} m_{21}}{E_{p p}}} .
\end{array}
$$

Here $\mathrm{f}$ is the neutrino magnetic moment in Bohr magnetons, $\mu_{\nu}=f \mu_{B}$, energies are in $\mathrm{eV}$ and $\mathrm{B}$ in Gauss. No information is provided from this analysis as to the location of this particular topology of the solar magnetic field. Helioseismology indicates that such a behaviour can only appear around the bottom of the convective zone at approximately $0.71 R_{S}$. This constrains, as seen from (35), the parameter $\Delta^{2} m_{21}$ to lie within less than an order of magnitude from $10^{-8} \mathrm{eV}^{2}$.

As far as ${ }^{8} \mathrm{~B}$ are concerned, their spread in energy is much larger than the previous ones 35

$$
0.814 \mathrm{MeV}<E_{8_{B}}<15 \mathrm{MeV}
$$

so some of their resonances overlap with the one from ${ }^{7} B e$. Given the survival probability expected for these neutrinos as derived in section 2

$$
0.35<P_{H}<0.63
$$

one may naively expect from (37), throughout most of their critical density range, $\gamma_{C}$ to be situated in the interval

$$
\gamma_{C} \simeq(0.15-0.30)
$$


However, due to the large energy spread here, no definite general criterion can be established for the field distribution along the critical density range. For a short interval at the beginning of this range owing to the overlap with the ${ }^{7} \mathrm{Be}$ resonance, the field should of course be constrained as discussed, but for the remainder, the actual profile is essentially unconstrained, except that the field should be substantially smaller on average. Only the total probability (12) can be used as an indicator, together with the requirement that the surface field should not exceed a few $\mathrm{kG}$. It may remain quite large over a considerable extent of the critical density range, so that an almost total conversion will occur for a substantial fraction of ${ }^{8} B$ neutrinos with $\gamma_{C}$ far exceeding in this zone the interval (49). Consequently, in the remaining part, the field should be much smaller with highly nonadiabatic transitions and $\gamma_{C}$ well below (49). Such is the case with one of the distributions to be examined below. So the requirement that $\gamma_{C}$ lies within the interval (49) for the resonances of ${ }^{8} B$ neutrinos is an indicative one for distributions varying smoothly in the range $x>x_{7 B e}$.

\subsection{Numerical Examples}

The general characteristics of suitable magnetic fields expressed in inequalities (45), (46) will now be confronted with specific field distributions. Equation (34) for the survival probability will be used together with the requirements $P_{I}<0.04, P_{L}>0.96$ and $0.35<$ $P_{H}<0.63$ as discussed in section 2 .

The starting point is a field spread only over the convective zone proposed years ago [44]. It changes from zero at $0.7 R_{S}$ to $10^{5} \mathrm{G}$ at $0.75 R_{S}$, then remains constant until $0.8 R_{S}$ and falls linearly to $2 \times 10^{3} \mathrm{G}$ at the surface of the Sun. For simplicity, a linear increase from $0.7 R_{S}$ will also be assumed:

$$
\begin{array}{lc}
B(x)=0 & 0 \leq x \leq 0.7 \\
B(x)=2 \times 10^{6}(x-0.7) & 0.7 \leq x \leq 0.75 \\
B(x)=10^{5} & 0.75 \leq x \leq 0.8 \\
B(x)=10^{5}-4.9 \times 10^{5}(x-0.8) & 0.8 \leq x \leq 1 .
\end{array}
$$

It is straightforward to realize that this field satisfies conditions (45), (46). Requiring in fact (45), (46) to hold simultaneously for the ${ }^{7} B e$ and all $p p$ resonances, i. e. up to $E_{p p_{\max }}=0.42 \mathrm{MeV}$, the intensity of the field in which $p p$ resonances are located is at most $1 / 7$ of the field at the ${ }^{7} \mathrm{Be}$ resonance. Since the distance between the critical densities corresponding to $E_{p p_{\max }}$ and $E_{7 B e}$ is $0.065 R_{S}$, (see eq. (35)), this leads, using (50), to

$$
x_{7 B e}-0.065 \leq 0.707
$$

(i. e. all $p p$ resonances should be located deeper than $0.707 R_{S}$ ) and

$$
x_{7 \mathrm{~B} e}>0.700,
$$

this last condition to ensure a non-vanishing magnetic field at the ${ }^{7} \mathrm{Be}$ resonance. Inequalities (51), (52) imply using (28)

$$
8.0 \times 10^{-9} \mathrm{eV}^{2} \leq \Delta^{2} m_{21} \leq 1.8 \times 10^{-8} \mathrm{eV}^{2} .
$$

This double inequality thus reflects the compatibility between the $p p$ and ${ }^{7} \mathrm{Be}$ solutions in (50) according to conditions (45), (46). No constraint from the ${ }^{8} B$ flux probability 
has yet been imposed. Using the lower bound of (53) in (45) with the corresponding critical density field $B\left(x_{7} B e\right)=10^{5} G$ to determine within this criterion the lowest neutrino magnetic moment compatible with $p p$ and ${ }^{7} B e$ solutions, one obtains

$$
\mu_{\nu} \geq 4.7 \times 10^{-12} \mu_{B}
$$

Using this value in eq. (34) for the survival probability with $P_{C}$ as in (24) one finds, however, a minor overlap in $\Delta^{2} m_{21}$ as regards $p p$ and ${ }^{7} B e$ neutrinos:

$$
\begin{array}{lll}
{ }^{8} \mathrm{~B} & \left(4.0-5.95 \times 10^{-9}\right) \mathrm{eV}^{2} & \left(9.0 \times 10^{-8}-1.25 \times 10^{-7}\right) \mathrm{eV}^{2} \\
{ }^{7} \mathrm{Be} & \left(1.2 \times 10^{-9}-1.13 \times 10^{-8}\right) \mathrm{eV}^{2} & \\
\text { pp } & <4.3 \times 10^{-11} \mathrm{eV}^{2} & >6.85 \times 10^{-9} \mathrm{eV}^{2}
\end{array}
$$

This overlap $\left[\Delta^{2} m_{21}=(0.69-1.1) \times 10^{-8} e V^{2}\right]$ is due to the fact that (45), (46) were imposed in this example for all $p p$ neutrinos which need not be the case. A small fraction of $p p$ neutrinos may have their resonances in a very short range with a field slightly in excess of $1 / 7$ of the field at the ${ }^{7} B e$ resonance, provided a substantial fraction of $p p$ resonances is located in a range where the field is much smaller. In terms of $x_{p p}$ this overlap range where (45), (46) are not satisfied is

$$
0.707<x_{p p}<0.721
$$

and for smaller $x_{p p}$ the field (50) is of course below $1 / 7$ of $B\left({ }^{7} B e\right)$. On the other hand the solution for ${ }^{8} B$ neutrinos in (55) is clearly inconsistent with the other two. It can be turned however consistent by relaxing (45), (46) over a short range, hence relaxing (54). In this way the set of solutions allowed by (50) may be investigated. The result is

$$
3.1 \times 10^{-12} \mu_{B} \leq \mu_{\nu} \leq 3.8 \times 10^{-12} \mu_{B}, 6.33 \times 10^{-9} \mathrm{eV}^{2} \leq \Delta^{2} m_{21} \leq 6.56 \times 10^{-9} \mathrm{eV}^{2}
$$

respectively. In order to have an idea of the amount of maximum deviation from (45), (46) involved, take the 'worst' solution, namely

$$
\mu_{\nu}=3.1 \times 10^{-12} \mu_{B} \quad, \quad \Delta^{2} m_{21}=6.33 \times 10^{-9} \mathrm{eV}^{2} .
$$

Condition (45) then gives $B\left(x_{7 B e}\right) \geq 133970 G$ (actually $B\left(x_{7 B e}\right)=10^{5} G$ ) while (46) is satisfied for all neutrinos with energies $E \leq 0.342 \mathrm{MeV}$. The field distribution (50), shown in fig. 2 , is therefore a borderline case in terms of providing a solution to the solar neutrino problem.

What one learns from this example is that imposing inequalities (45), (46) over the whole $p p$ resonance range provides an approximate sufficient condition for a suitable field distribution. Minor deviations may be allowed for some $x_{p p}$, since these inequalities were derived from the simplified formula (37) and a fixed neutrino energy. In any case, though, the main characteristic of a field increasing by a factor $>6$ over a fraction shorter than $9 \%$ of the solar radius seems essential, in order for the field to be compatible with solar neutrino data: the smearing effect due to the energy distribution is not as large with $p p$ as with ${ }^{8} B$ neutrinos, so this criterion should be retained.

Let us reverse the attitude and search for a field distribution satisfying (45), (46) behaving linearly with $x$ for simplicity. The ${ }^{7} \mathrm{Be}$ resonance is assumed to be located at the bottom of the convective zone where the field is taken to be maximal $\left(x_{B e}=0.71\right.$, 
$\left.\Delta^{2} m_{21}=1.6 \times 10^{-8} \mathrm{eV}^{2}\right)$. It then falls to $2 \times 10^{3} G$ at the surface of the Sun. Taking $f=10^{-12}$, one has $B\left(x^{7} B e\right) \geq 6.6 \times 10^{5} G$ and $(46)$ becomes

$$
B\left(x_{p p}\right) \leq 6.11 \times 10^{7} \frac{1}{\sqrt{E_{p p}}} G
$$

with $E_{p p}$ given in $\mathrm{eV}$. Given the fact that $p p$ resonances start at 0.065 below $x_{7^{7} B e}$, one has essentially two kinds of options:

1. First option. One imposes inequality (46) to hold only for part of $p p$ resonances $\left(x_{p p} \leq x_{7_{B}}-\Delta x\right)$ thus allowing for its violation in the interval $x_{7_{B e}}-\Delta x \leq x_{p p} \leq x_{7_{B e}}-$ 0.065. Consequently $B(x)$ has to further decrease inwards from its value at $x_{p p}=x_{7_{B e}}-$ $\Delta x$ in order to compensate for excessive neutrino conversion in this interval. (Recall that $p p$ neutrinos are practically unconverted). So, taking for instance $\Delta x=0.09$, the corresponding energy $E_{p p}$ in (46) for $\Delta^{2} m_{21}=1.6 \times 10^{-8} \mathrm{eV}^{2}$ is $3.17 \times 10^{5} \mathrm{eV}$. This gives $B\left(x_{p p}\right) \leq 1.08 \times 10^{5} G$ from $(59)\left(B\left(x_{p p}\right) \leq 9.4 \times 10^{4} G\right.$ if one required all $p p$ neutrinos to satisfy (46), i. e. $\Delta x=0.065)$. Hence, choosing $B\left(x_{p p}\right) \leq 9.4 \times 10^{4} G$ to allow for an overlap, the field will be

$$
\begin{array}{lc}
B(x)=0 & x \leq 0.606 \\
B(x)=6.73 \times 10^{6}(x-0.62)+9.4 \times 10^{4} & 0.606 \leq x \leq 0.71 \\
B(x)=-2.407 \times 10^{6}(x-0.71)+7 \times 10^{5} & 0.71 \leq x \leq 1
\end{array}
$$

However the probability constraints on $P_{H}, P_{I}, P_{L}$ give respectively

$$
\begin{array}{lll}
{ }^{8} \mathrm{~B} & (4.8-6.8) \times 10^{-9} \mathrm{eV}^{2} & (1.95-2.70) \times 10^{-7} \mathrm{eV}^{2} \\
{ }^{7} \mathrm{Be} & \left(1.12 \times 10^{-9}-2.1 \times 10^{-8}\right) \mathrm{eV}^{2} & \\
p p & <8.5 \times 10^{-11} \mathrm{eV}^{2} & 1.1 \times 10^{-8} \mathrm{eV}^{2}
\end{array}
$$

with $\mu_{\nu}=10^{-12} \mu_{B}$, showing an inconsistency with ${ }^{8} B$. This is solved by requiring a more moderate slope of the field along the outer layers of the convective zone. A decrease from $2.2 \times 10^{5} G$ to $2 \times 10^{4} G$ at $x=0.91$ is sufficient while keeping the same values at the surface and $x_{7} B e=0.71$. Thus the same suppression of ${ }^{8} B$ neutrinos is achieved at larger densities than those corresponding to the interval $(4.8-6.8) \times 10^{-9} \mathrm{eV}^{2}$ in (61). The field is then (see fig.3):

$$
\begin{array}{lc}
B(x)=0 & x \leq 0.606 \\
B(x)=6.73 \times 10^{6}(x-0.62)+9.4 \times 10^{4} & 0.606 \leq x \leq 0.71 \\
B(x)=-3.4 \times 10^{6}(x-0.71)+7 \times 10^{5} & 0.71 \leq x \leq 0.91 \\
B(x)=-2 \times 10^{5}(x-0.91)+2 \times 10^{4} & 0.91 \leq x \leq 1
\end{array}
$$

with a solution $\left(f=10^{-12}\right)$ Q

$$
\begin{array}{lll}
{ }^{8} B & (1.37-1.90) \times 10^{-8} \mathrm{eV}^{2} & (1.95-2.70) \times 10^{-7} \mathrm{eV}^{2} \\
{ }^{7} \mathrm{Be} & \left(3.2 \times 10^{-9}-2.1 \times 10^{-8}\right) \mathrm{eV}^{2} & \\
{ }_{p p} & <1.34 \times{ }^{-10} \mathrm{eV}^{2} & >1.65 \times 10^{-8} \mathrm{eV}
\end{array}
$$

Hence $\Delta^{2} m_{21}=(1.65-1.90) \times 10^{-8} \mathrm{eV}^{2}$.

\footnotetext{
${ }^{2}$ It is worth noting that the range of possible neutrino magnetic moments extends in this case down to $\mu_{\nu}=6.5 \times 10^{-13} \mu_{B}$.
} 
2. Second option. Inequality (46) is imposed as from $x_{7} B e-0.065$ inwards. $\mathrm{B}(\mathrm{x})$ may then ramain constant below this value since it will be sufficiently small throughout all $x_{p p}$ to ensure enough neutrino survival. Again a large slope at large $x$ (upper convective zone) must be avoided for consistency with ${ }^{8} B$ neutrinos. So $B\left(x_{p p}\right)=9.4 \times 10^{4} G$ for all $x_{p p}$ and, as before, $B\left(x_{7} B e\right)=7 \times 10^{5} G$, (see (fig.4)):

$$
\begin{array}{lc}
B(x)=9.4 \times 10^{4} & x \leq 0.645 \\
B(x)=9.32 \times 10^{6}(x-0.645)+9.4 \times 10^{4} & 0.645 \leq x \leq 0.71 \\
B(x)=-3.4 \times 10^{6}(x-0.71)+7 \times 10^{5} & 0.71 \leq x \leq 0.91 \\
B(x)=-2 \times 10^{5}(x-0.91)+2 \times 10^{4} & 0.91 \leq x \leq 1
\end{array}
$$

with a solution $\left(f=10^{-12}\right)$

$$
\begin{array}{lll}
{ }^{8} \mathrm{~B} & (1.37-1.91) \times 10^{-8} \mathrm{eV}^{2} & (1.73-2.37) \times 10^{-8} \mathrm{eV}^{2} \\
{ }^{7} \mathrm{Be} & \left(3.2 \times 10^{-9}-1.95 \times 10^{-8}\right) \mathrm{eV}^{2} & \\
p p & <1.34 \times 10^{-10} \mathrm{eV}^{2} & >1.5 \times 10^{-8} \mathrm{eV}^{2} .
\end{array}
$$

The range of solutions is slightly enlarged here relative to option $1: \Delta^{2} m_{21}=(1.50-$ 1.91) $\times 10^{-8} \mathrm{eV}^{2}$.

Such a large field at the bottom of the convective zone $\left(7 \times 10^{5} G\right)$ is a consequence of requiring a comparatively small value of the neutrino magnetic moment $\left(\mu_{\nu}=10^{-12} \mu_{B}\right)$ and, especially, the ${ }^{7} \mathrm{Be}$ resonance to to lie at $0.71 R_{S}$ instead of lower densities as in the previous example (50). For lower densities, adiabaticity is in fact satisfied with lower magnetic fields (see eq. (25)).

At this stage it should have become obvious that a monotonically decreasing field from the core or the inner radiative zone to the solar surface such as the ones proposed in refs. 41, 42 must be ruled out in the light of solar neutrino data. Also linearly varying profiles, although not to be excluded, can only provide borderline solutions.

To conclude this section, four more field profiles rising sharply at the bottom of the convective zone will be briefly reviewed. The first two are variants of one of the fields proposed in ref. 43] namely,

$$
B(x)=\frac{3.048 \times 10^{4}}{\operatorname{ch}[20(x-0.71)]} G \quad, \quad 0.71 \leq x \leq 1
$$

with $B=2.8 \times 10^{3} G$ and $B=0$ for $x<0.71$ (see fig.5). For the first of these one obtains the following two limiting cases

$$
\begin{array}{lll}
{ }^{8} B & (1.60-2.50) \times 10^{-8} \mathrm{eV}^{2} & (1.10-1.54) \times 10^{-7} \mathrm{eV}^{2} \\
{ }^{7} \mathrm{Be} & \left(5.2 \times 10^{-9}-1.6 \times 10^{-8}\right) \mathrm{eV}^{2} & \\
p p & <7.4 \times 10^{-11} \mathrm{eV}^{2} & >1.43 \times 10^{-8} \mathrm{eV}^{2}
\end{array}
$$

with $\mu_{\nu}=3.4 \times 10^{-11} \mu_{B}$ and

$$
\begin{array}{lll}
{ }^{8} B & (1.50-2.40) \times 10^{-8} \mathrm{eV}^{2} & (1.10-1.55) \times 10^{-7} \mathrm{eV}^{2} \\
{ }^{7} \mathrm{Be} & \left(4.9 \times 10^{-9}-1.6 \times 10^{-8}\right) \mathrm{eV}^{2} & \\
\text { pp } & <6.9 \times 10^{-11} \mathrm{eV}^{2} & >1.60 \times 10^{-8} \mathrm{eV}^{2}
\end{array}
$$

with $\mu_{\nu}=3.65 \times 10^{-11} \mu_{B}$. This leads to the range of solutions:

$$
3.4 \times 10^{-11} \mu_{B} \leq \mu_{\nu} \leq 3.65 \times 10^{-11} \mu_{B}, 1.55 \times 10^{-8} \mathrm{eV}^{2} \leq \Delta^{2} m_{21} \leq 1.60 \times 10^{-8} \mathrm{eV}
$$


with (46) satisfied for $x_{p p} \leq x_{7 B e}-0.082$. As opposed to the previous examples, the field is constant below its maximum, so the fraction of $p p$ resonances that do not satisfy (46) has to be smaller. For the second of distributions (66), with the field limited to the convection zone, the range of solutions necessarily extends. In fact the two limiting cases are now

$$
\begin{array}{lll}
{ }^{8} B & (1.60-2.50) \times 10^{-8} \mathrm{eV}^{2} & (1.0-1.42) \times 10^{-7} \mathrm{eV}^{2} \\
{ }^{7} \mathrm{Be} & \left(5.1 \times 10^{-9}-1.60 \times 10^{-8}\right) \mathrm{eV}^{2} & \\
p p & <6.4 \times 10^{-11} \mathrm{eV}^{2} & >7.3 \times 10^{-9} \mathrm{eV}^{2}
\end{array}
$$

with $\mu_{\nu}=3.4 \times 10^{-11} \mu_{B}$ and

$$
\begin{array}{lll}
{ }^{8} \mathrm{~B} & (3.3-7.3) \times 10^{-9} \mathrm{eV}^{2} & (1.10-1.44) \times 10^{-7} \mathrm{eV}^{2} \\
{ }^{7} \mathrm{Be} & \left(2.0 \times 10^{-9}-1.6 \times 10^{-8}\right) \mathrm{eV}^{2} & \\
\text { pp } & \text { no solution } & >7.3 \times 10^{-9} \mathrm{eV}^{2}
\end{array}
$$

with $\mu_{\nu}=1.3 \times 10^{-10} \mu_{B}$. The range of solutions is now

$$
3.4 \times 10^{-11} \mu_{B} \leq \mu_{\nu} \leq 1.3 \times 10^{-10} \mu_{B}, 7.3 \times 10^{-9} \mathrm{eV}^{2} \leq \Delta^{2} m_{21} \leq 1.60 \times 10^{-8} \mathrm{eV}^{2} .
$$

The last two profiles to be examined bear no discontinuity. They are

$$
\begin{array}{ccc}
B(x)=2.16 \times 10^{3} G & x \leq 0.7105 \\
B(x)=8.7 \times 10^{4}\left[1-\left(\frac{x-0.75}{0.04}\right)^{2}\right] G & 0.7105 \leq x \leq 0.7483
\end{array}
$$

with

$$
B(x)=10^{5}[1-3.4412(x-0.71)] G \quad 0.7483 \leq x \leq 1
$$

and

$$
B(x)=\frac{8.684 \times 10^{4}}{\operatorname{ch}[20(x-0.7483)]} G \quad 0.7483 \leq x \leq 1
$$

shown in figs. 6 and 7 respectively. The first of these has a very limited range of solutions of which some violate up to $30 \%$ the inequality (45). Also (46) is satisfied only for $E<0.381 \mathrm{MeV}$, a similar situation to that encountered with the linear field distribution (50). Again, this is a borderline case and the limiting solutions are

$$
\begin{array}{lll}
{ }^{8} \mathrm{~B} & (6.2-9.1) \times 10^{-9} \mathrm{eV}^{2} & \left(8.6 \times 10^{-8}-1.2 \times 10^{-7}\right) \mathrm{eV}^{2} \\
{ }^{7} \mathrm{Be} & (3.5-6.5) \times 10^{-9} \mathrm{eV}^{2} & \\
p p & <1.0 \times 10^{-10} \mathrm{eV}^{2} & >6.5 \times 10^{-9} \mathrm{eV}^{2}
\end{array}
$$

with $\mu_{\nu}=4.1 \times 10^{-12} \mu_{B}$ and

$$
\begin{array}{lll}
{ }^{8} \mathrm{~B} & (4.8-6.8) \times 10^{-9} \mathrm{eV}^{2} & \left(9.5 \times 10^{-8}-1.3 \times 10^{-7}\right) \mathrm{eV}^{2} \\
{ }^{7} \mathrm{Be} & \left(1.3 \times 10^{-9}-1.25 \times 10^{-8}\right) \mathrm{eV}^{2} & \\
\text { pp } & <9.2 \times 10^{-11} \mathrm{eV}^{2} & >6.9 \times 10^{-9} \mathrm{eV}^{2}
\end{array}
$$

with $\mu_{\nu}=6.15 \times 10^{-12} \mu_{B}$. These lead to the following range of solutions

$$
4.1 \times 10^{-12} \mu_{B} \leq \mu_{\nu} \leq 6.15 \times 10^{-12} \mu_{B}, 6.5 \times 10^{-9} e V^{2} \leq \Delta^{2} m_{21} \leq 6.9 \times 10^{-9} \mathrm{eV}^{2} .
$$


In contrast to $(73),(74)$, the second combination $(73),(75)$, provides a much larger set of solutions whose limiting cases are

$$
\begin{array}{lll}
{ }^{8} \mathrm{~B} & (1.29-2.1) \times 10^{-8} \mathrm{eV}^{2} & \left(9.5 \times 10^{-8}-1.3 \times 10^{-7}\right) \mathrm{eV}^{2} \\
{ }^{7} \mathrm{Be} & \left(4.33 \times 10^{-9}-1.32 \times 10^{-8}\right) \mathrm{eV}^{2} & \\
p p & <5.6 \times 10^{-11} \mathrm{eV}^{2} & >6.8 \times 10^{-9} \mathrm{eV}^{2}
\end{array}
$$

for $\mu_{\nu}=7.2 \times 10^{-12} \mu_{B}$ and

$$
\begin{array}{lll}
{ }^{8} \mathrm{~B} & (3.30-7.32) \times 10^{-9} \mathrm{eV}^{2} & (1.0-1.4) \times 10^{-7} \mathrm{eV}^{2} \\
{ }^{7} \mathrm{Be} & \left(1.85 \times 10^{-9}-1.51 \times 10^{-8}\right) \mathrm{eV}^{2} & \\
\text { pp } & \text { no solution } & >7.32 \times 10^{-9} \mathrm{eV}^{2}
\end{array}
$$

for $\mu_{\nu}=2.1 \times 10^{-11} \mu_{B}$, shown in fig.8. Hence, for the field (73), (75), the set of solutions is

$$
7.2 \times 10^{-12} \mu_{B} \leq \mu_{\nu} \leq 2.1 \times 10^{-11} \mu_{B}, 7.3 \times 10^{-9} \mathrm{eV}^{2} \leq \Delta^{2} m_{21} \leq 1.3 \times 10^{-8} \mathrm{eV}^{2}
$$

The distinguishing feature between (74) and (75) is the same as between field profiles (60), (62): two fall offs along the convective zone, one linear and the other with an upward facing concavity. The second type is clearly favoured while the first is disfavoured. A downward facing concavity along the convective zone thus seems to be excluded. The type of exponential fall off proposed years ago [42] where this downward concavity predominates, namely (see fig.8),

$$
B(x)=\frac{B_{0}}{1+\exp \frac{x-0.95}{0.01}} \quad, \quad 0.7483 \leq x \leq 1
$$

is also not possible. The main reason is that the moderate suppression required for ${ }^{8} B$ neutrinos demands their resonances to be mainly located in a zone where the field is substantially smaller than at the ${ }^{7} B e$ resonance. But then the $p p$ resonances are 'dragged' into a zone where the field is far too large.

The main requirements for a suitable field, as from the analysis of this section are therefore:

1) A very sharp rise across the bottom of the convective zone by at least a factor of 6 - 7 over a distance not greater than approximately $9 \%$ of the solar radius. This sharp increase may be a discontinuity.

2) A decrease along the convective zone whose slope is at first strong and then reduces to become very moderate or even closer to constant as one approaches the surface.

\section{Outlook and Conclusions}

The solar neutrino data were used to obtain information or, possibly, 'derive' the likely profile of the solar magnetic field on the basis of the existence of a neutrino magnetic moment and the RSFP mechanism. The main point of the analysis relies on associating neutrino energy with resonance location within the Sun. The experimental data and general basic features of solar models overwhelmingly favour an energy dependent neutrino suppression, the main uncertainty left being the amount of suppression of the highest 
energetic ${ }^{8} B$ neutrinos. So neutrino suppression is likely to be correlated to resonance location within the Sun and thus to the profile of the field intensity, since a strong field at the resonance is responsible for a high transition rate. These are the underlying arguments for a possible tomography of the solar magnetic field.

On the basis of these arguments, the large suppression of the intermediate energy neutrinos consisting mainly of the ${ }^{7} B e$ line at $E=0.86 \mathrm{MeV}$, along with the total or nearly total survival of the detected $p p$ neutrinos $\left(0.236 \mathrm{MeV}<E_{p p}<0.42 \mathrm{MeV}\right)$ is a clear indication of a solar field rising sharply along a relatively short distance. Such a topology can only be expected to be located around the bottom of the convective zone within a layer of at most $(9-10) \%$ of the solar radius. From the analysis of the previous section the field should rise by a factor of at least $6-7$. All is consistent with this rise being extremely sudden, even in the form of a discontinuity, or to span most of that layer. If one believes in the RSFP mechanism, one may get an upper bound on the field (see eq.(46)) from the inner edge of this zone down to approximately $0.59 R_{S}$ to be $B_{\max } \simeq(1.1-1.3) \times 10^{4} G$, taking $\mu_{\nu}=10^{-11} \mu_{B}$. At the peak the field should be $B_{\min } \simeq 7 \times 10^{4} G$ for the same $\mu_{\nu}$. Both these bounds are inversely proportional to the neutrino magnetic moment. Deeper into the radiation zone the field is essentially unknown, although there is no ground to believe in other large spatial variations.

As regards the convective zone, one expects a decreasing field with no sudden variations, the analysis favouring a larger slope towards the bottom with a more moderate one towards the surface. The 'true' field profile thus appears to be consistent with any of the ones shown in figs. 2 through 7 , with fig. 8 excluded because of the shape of the field decrease along the convective zone.

In the original papers [ [I] introducing the hypothetical anticorrelation of active neutrino flux with solar activity as evidenced through the sunspots, it was suggested that the sunspot activity effect could extend as deep as the bottom of the convective zone. This is however by no means clear, hence the extent of neutrino trajectory affected by this 11 year sunspot cycle is unknown. The evaluation of the adiabaticity parameter $\gamma_{C}$ (eq.(25)) for all magnetic field distributions considered shows that only the one in fig.5 (eq. (66) with $B=0$ for $x<0.71$ ) may generate moderately adiabatic transitions for the Super-Kamiokande neutrinos with energies ranging from their $7 \mathrm{MeV}$ threshold up to $9 \mathrm{MeV}$. Even this situation will only occur at the upper edge of the solution, namely $\mu_{\nu}=1.3 \times 10^{-10} \mu_{B}$, $\Delta^{2} m_{21}=1.6 \times 10^{-8} \mathrm{eV}^{2}$, (eq. (72)), in clear conflict with the astrophysical bounds on $\mu_{\nu}$ [45]. For all other cases the Super-Kamio-kande neutrinos are expected to undergo nonadiabatic transitions in the Sun, so no anticorrelation with sunspot activity is expected, even if the effect extends deep down into the convective zone. For the Chlorine experiment on the other hand, a larger fraction of ${ }^{8} B$ neutrinos may be anticorrelated, since the energy threshold is much lower, but the effect may be totally unclear due to the dominant influence of the more energetic neutrinos whose resonances are non-adiabatic. Therefore RSFP scenarios are fully consistent with the absence of anticorrelation in the experiments so far [15].

As far as the approach used throughout this paper is concerned, a few words must be added regarding the simplified treatment of the intermadiate energy neutrinos. An overall suppression factor of at least $96 \%$ was considered for all of them, with a fixed energy $E=E_{7_{B e}}=0.86 \mathrm{MeV}$. They however span an energy range up to $1.7 \mathrm{MeV}$ with this ${ }^{7} \mathrm{Be}$ line accounting for the largest part $(\simeq 71 \%)$. Nevertheless, a more elaborate procedure is hardly justified at the present stage of both data and theoretical models and 
should not change significantly the results derived here. In fact the suppression peak will necessarily be still assumed to occur at the $0.86 \mathrm{MeV}{ }^{7} \mathrm{Be}$ line, with the other ${ }^{7} \mathrm{Be}$ line at $0.38 \mathrm{MeV}$, immersed in the $p p$ energy range, being naturally much less suppressed. The CNO continuum may also suffer this suppression peak around $0.86 \mathrm{MeV}$ but, for the remainder, it need not, along with the pep line $(E=1.442 \mathrm{MeV})$, undergo a large suppression.

It should be stressed that all information that can be obtained from the RSFP on the solar magnetic field only concerns its profile and not its absolute magnitude because the order parameter of the analysis is the product $\mu B$. The analysis and results refer only to the transverse component of the magnetic field along the direction of propagation and nothing can be inferred as to its latitude dependence. They necessarily bear the uncertainties common to all solar physics models.

From the experimental side, more abundant statistics and precise data together with new solar neutrino experiments are essential in order to improve our knowledge, along with independent laboratory searches for neutrino magnetic moment effects. In has been shown in this paper that the necessary $\mu_{\nu}$ to provide a solution to the solar neutrino problem, thereby leading to information on the magnetic field, is within most of the relatively stringent astrophysical bounds [45]. Although this lies almost 6 orders of magnitude above the electroweak standard model prediction, one should keep in mind that the standard model itself is full of fine tuning and similarly large hierarchy problems affecting for instance fermion masses. Furthermore, there exist successful models [46] for large $\mu_{\nu}$ which do not conflict with the necessary smallness of neutrino mass.

\section{Acknowledgements}

The author wishes to express his gratitude to John Ralston for enlightening discussions and to the Department of Physics and Astronomy of the University of Kansas for hospitality and financial support. He is also grateful to Stanley Brodsky and the SLAC Theory Group for providing a visitorship during which part of this work was performed.

\section{References}

[1] R. Davis, D. S. Harmer, K. C. Hoffman, Phys. Rev. Lett. 20 (1968) 1205.

[2] S. Degl'Innocenti et al., Astrop. Phys. 7 (1997) 77.

[3] A. Cisneros, Astrophys. Space Sci. 10 (1971) 87.

[4] M. B. Voloshin, M. I. Vysotsky, L. B. Okun, Sov. J. Nucl. Phys. 44 (1986) 440; M. B. Voloshin, M. I. Vysotsky, Sov. J. Nucl. Phys. 44 (1986) 544.

[5] J. K. Rowley, B. T. Cleveland, R. Davis, Am. Inst. of Phys. Conf. Proc. 126 (1985) 1; R. Davis, 7th Workshop on Grand Unification: ICOBAN '86, ed. J. Arafune; R. Davis. B. T. Cleveland, J. K. Rowley, Proc. of 20th Int. Cosmic Ray Conf., Moscow 19874 (1987) 328.

[6] J. N. Bahcall, G. B. Field, W. H. Press, Ap. J. 320 (1987) L69. 
[7] B. W. Filippone. P. Vogel, Phys. Lett. B246 (1990) 546.

[8] J. W. Bieber et al., Nature 348 (1990) 407.

[9] X. Shi, D. N. Schramm, R. Rosner, D. S. Dearborn, Comments Nucl. Part. Phys. 21 (1993) 151.

[10] Homestake Collaboration: K. Lande in Proc. of Neutrino'96, Eds. K. Enqvist, K. Huitu, J. Maalampi, World Scientific 1997, p.25.

[11] Kamiokande Collaboration: Y. Suzuki, ibid., p.73.

[12] SAGE Collaboration: V. N. Gavrin, ibid., p.14.

[13] Gallex Collaboration: M. Cribier, in TAUP'97, 5th International Workshop on Topics in Astroparticle and Underground Physics, Gran Sasso, Assergi, Italy, 1997, to appear in the Proceedings.

[14] SuperKamiokande Collaboration: J. Hill in Proc. of the XXXII Rencontre de Moriond (Jan. 1997), to be published.

[15] G. Walther, Phys. Rev. Lett. 79 (1998) 4522.

[16] S. Turck-Chieze, I. Lopes, Ap. J. 408 (1993) 347.

[17] S. Turck-Chieze et al. Ap. J. 335 (1988) 415.

[18] C. R. Proffit, Ap. J. 425 (1994) 849.

[19] O. Richard et al., Astron. Astrophys. 312 (1996) 1000.

[20] S. Degl'Innocenti et al., Astron. Astrophys. Suppl. Ser. 123 (1997) 1.

[21] J. N. Bahcall, M. H. Pinsonneault, Rev. Mod. Phys. 67 (1995) 781.

[22] J. N. Bahcall in Proc. of Neutrino'96, Eds. K. Enqvist, K. Huitu, J. Maalampi, World Scientific, 1997, p. 56.

[23] L. Wolfenstein, Phys. Rev. D17 (1978) 2369; D20 (1979) 2364; S. P. Mikheyev, A. Smirnov, Sov. J. Nucl. Phys. 42 (1985) 913.

[24] P. F. Harrison et al., Phys. Lett. B374 (1996) 111.

[25] C. S. Lim, W. J. Marciano, Phys. Rev. D37 (1988) 1368; E. K. Akhmedov, Phys. Lett. B213 (1988) 64; E. K. Akhmedov, Sov. J. Nucl. Phys. 48 (1988) 42.

[26] E. Roulet, Phys. Rev. D44 (1991) R935; M. M. Guzzo, A. Masiero, S. T. Petcov, Phys. Lett. B260 (1991) 154; J. Pulido, Mod. Phys. Lett. A8 (1993) 1273; P. I. Krastev and J. N. Bahcall, astro-ph/9703267, talk given at Symposium on Flavour Changing Neutral Currents: Present and Future Studies (FCNC 97), Santa Monica, CA, 19-21 Feb. 1997.

[27] W. Kwong, S. P. Rosen, Phys. Rev. Lett. 73 (1994) 369. 
[28] P. I. Krastev, S. T. Petcov, Phys. Lett. B395 (1997) 69.

[29] J. N. Bahcall, Phys. Lett. B338 (1994) 276.

[30] L. Wolfenstein, P. I. Krastev, Phys. Rev. D55 (1997) 4405.

[31] K. M. Heeger, R. G. H. Robertson, Phys. Rev. Lett. 77 (1996) 3720.

[32] V. Castellani et al. Phys. Rep. 281 (1997) 309.

[33] V. Berezinsky, Comm. Nucl. Part. Phys., 21 (1994) 249.

[34] Review of Particle Properties, R. M. Barnett et al. Phys. Rev. D54 (1996) 1.

[35] J. N. Bahcall, R. K. Ulrich, Rev. Mod. Phys. 60 (1988) 297.

[36] L. Landau, Phys. Z. Sowietunion 21932 46; C. Zener, Proc. R. Society, A137 (1932) 696; E. C. G. Stueckelberg, Helv. Phys. Acta 5 (1932) 369.

[37] J. Pulido, Phys. Rep. 211 (1992) 167.

[38] A. Dar, G. Shaviv, Ap. J. 468 (1996) 933.

[39] S. J. Parke, Phys. Rev. Lett. 57 (1996) 1275.

[40] J. Pulido, Phys. Rev. D48 (1993) 1492.

[41] E. K. Akhmedov, O, V. Bychuk, Sov. Phys. JETP 68 (1989) 250.

[42] E. K. Akhmedov, A. Lanza, S. T. Petcov, Phys. Lett. 303 (1993) 85.

[43] E. K. Akhmedov, A. Lanza, S. T. Petcov, Phys. Lett. 348 (1995) 124.

[44] A. B. Balantekin, P. J. Hatchell, F. Loreti, Phys. Rev. D41 (1990) 3583.

[45] S. Nussinov. Y. Rephaeli, Phys. Rev. D36 (1987) 2278; I. Goldman et al., Phys. Rev. Lett. 60 (1988), G. Lattimer, J. Cooperstein, Phys. Rev. Lett. 61 (1988) 23; R. Barbieri, R. N. Mohapatra, Phys. Rev. Lett. 61 (1988) 27; D. Notzold, Phys. Rev. D38 (1988) 1658.

[46] S. M. Barr, E. M. Freire, A. Zee, Phys. Rev. Lett. 65 (1990) 2626. 


\begin{tabular}{cccc}
\hline \hline Experiment & Ref. & Data & Units \\
\hline Homestake & {$[10]$} & $2.54 \pm 0.14 \pm 0.14$ & SNU \\
Kamiokande & {$[11$} & $2.80 \pm 0.19 \pm 0.33$ & $10^{6} \mathrm{~cm}^{-2} \mathrm{~s}^{-1}$ \\
SAGE & {$[12$} & $72 \pm_{10}^{12} \pm_{7}^{5}$ & SNU \\
Gallex & {$[13$} & $76.2 \pm 6.5 \pm 5$ & SNU \\
SuperKamiokande & {$[14]$} & $2.51 \pm_{0.13}^{0.14}$ & $10^{6} \mathrm{~cm}^{-2} \mathrm{~s}^{-1}$ \\
\hline \hline
\end{tabular}

Table I. Neutrino event rates measured by solar neutrino experiments.

\begin{tabular}{|c|c|c|c|c|c|c|}
\hline & TCL《16] & 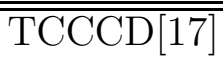 & P94[18] & "RVCD96 [19 & FRANEC96[20] & BP95[21] \\
\hline \multicolumn{7}{|c|}{ Chlorine } \\
\hline $\mathrm{pp}$ & 0.0 & 0.0 & 0.0 & 0.0 & 0.0 & 0.0 \\
\hline pep & 0.22 & 0.21 & 0.222 & 0.221 & 0.224 & 0.224 \\
\hline${ }^{7} \mathrm{Be}$ & 1.10 & 0.995 & 1.24 & 1.15 & 1.08 & 1.24 \\
\hline${ }^{13} \mathrm{~N}$ & 0.063 & 0.104 & 0.109 & 0.095 & 0.0901 & 0.105 \\
\hline${ }^{15} \mathrm{O}$ & 0.21 & 0.37 & 0.379 & 0.327 & 0.306 & 0.371 \\
\hline${ }^{8} \mathrm{~B}$ & 4.63 & 4.06 & 7.19 & 7.03 & 5.73 & 7.35 \\
\hline total & 6.23 & 5.75 & 9.1 & 8.8 & 7.4 & 9.3 \\
\hline \multicolumn{7}{|c|}{ Gallium } \\
\hline $\mathrm{pp}$ & 71.1 & 70.6 & 69.7 & 70.1 & 70.7 & 69.7 \\
\hline pep & 2.99 & 2.795 & 2.99 & 2.97 & 3.01 & 3.01 \\
\hline${ }^{7} \mathrm{Be}$ & 30.9 & 30.6 & 37.9 & 35.1 & 32.9 & 37.7 \\
\hline${ }^{13} \mathrm{~N}$ & 2.36 & 3.87 & 3.95 & 3.45 & 3.27 & 3.82 \\
\hline${ }^{15} \mathrm{O}$ & 0.21 & 6.5 & 6.46 & 5.58 & 5.22 & 6.32 \\
\hline${ }^{8} \mathrm{~B}$ & 10.77 & 9.31 & 15.7 & 15.4 & 12.5 & 16.1 \\
\hline total & 122.5 & 124 & 137 & 133 & 128 & 137 \\
\hline \multicolumn{7}{|l|}{ Kam } \\
\hline & 4.4 & 3.8 & 6.48 & 6.33 & 5.16 & 6.62 \\
\hline
\end{tabular}

Table II. Contributions of different sources of neutrinos to total capture rates in the Chlorine, Kamiokande and Gallium experiments from each standard solar model considered. All models include diffusion except for TCL [16] and TCCCD [17]. Units are $10^{6} \mathrm{~cm}^{-2} \mathrm{~s}^{-1}$ for Kamiokande and SNU for Chlorine and Gallium. 
Fig.1 Survival probability of $p p$ neutrinos with a magnetic moment $\mu_{\nu}=1.3 \times 10^{-10} \mu_{B}$ for the field distribution given by eq.(66) with $B=0$ in the radiative zone and core. The left end of the diagram corresponds to the resonance being close to the surface of the Sun or in the vacuum where the field is negligible, whereas the right end corresponds to a resonance in the interior (radiative zone and core) where the field also vanishes.

Fig.2 Field distribution given by eq.(50). Coordinate $x$ denotes the fraction of the Sun's radius and the magnetic field is given in Gauss. The solution range is given by eq. (57).

Fig.3 Field distribution given by eq.(62) ('option 1'). For the solution see eq.(63).

Fig.4 Field distribution given by eq.(64) ('option 2'). For the solution see eq.(65).

Fig.5 Field distribution given by eq.(66) with $B=0$ below the convective zone. The range of solutions is greatly extended relative to previous cases (see eq.(72)) because the rise in the field at the bottom of the convective zone is both very large and sudden so that inequality (45) is largely satisfied. Also from the bottom of the convective zone to the solar surface the field has the 'adequate' shape that can account for a large range in terms of the ${ }^{8} B$ solution.

Fig.6 Field distribution given by eqs.(73), (74). The range of solutions is very limited (see eq.(78)).

Fig.7 Field distribution given by eqs.(73), (75). Although the magnitude of the field is no larger than in the previous case, the solution is greatly extended (compare eq. (78) with eq.(81)) owing to the difference in shapes.

Fig.8 Field distribution given by eq.(82). Although this distribution has the correct shape across the bottom of the convective zone with a large and sharp increase, it fails to provide a solution, owing to its shape along the convective zone. 


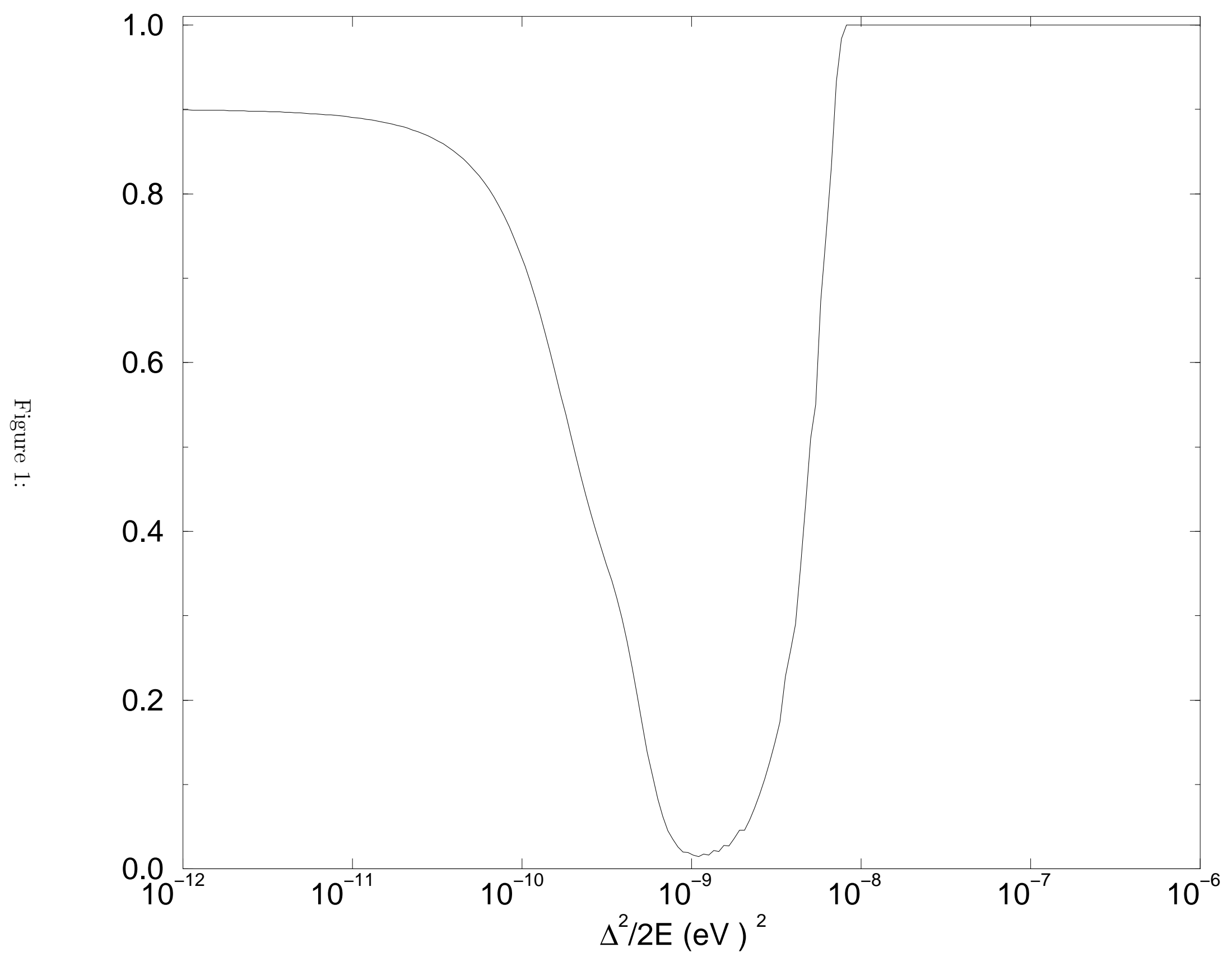




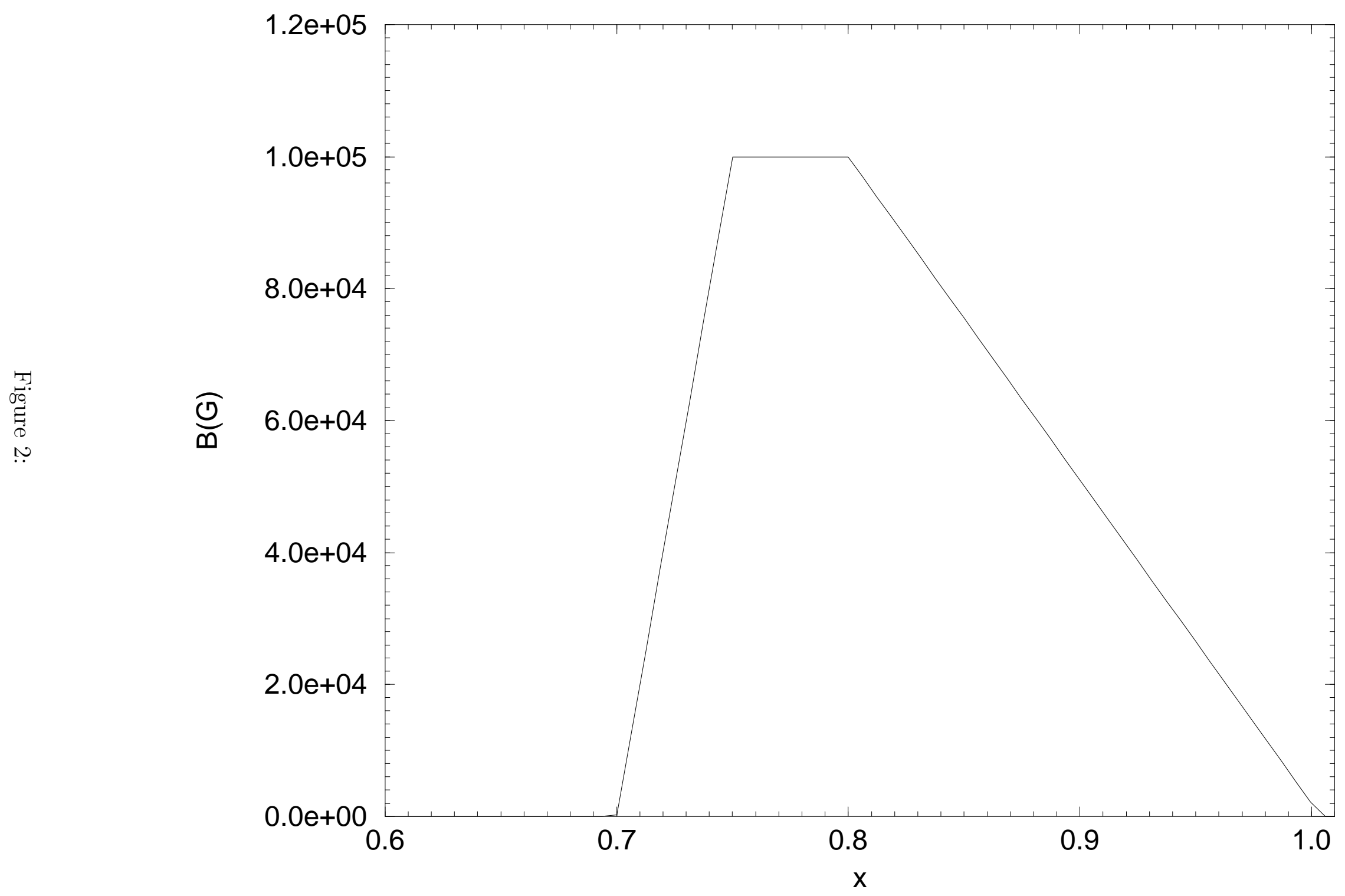




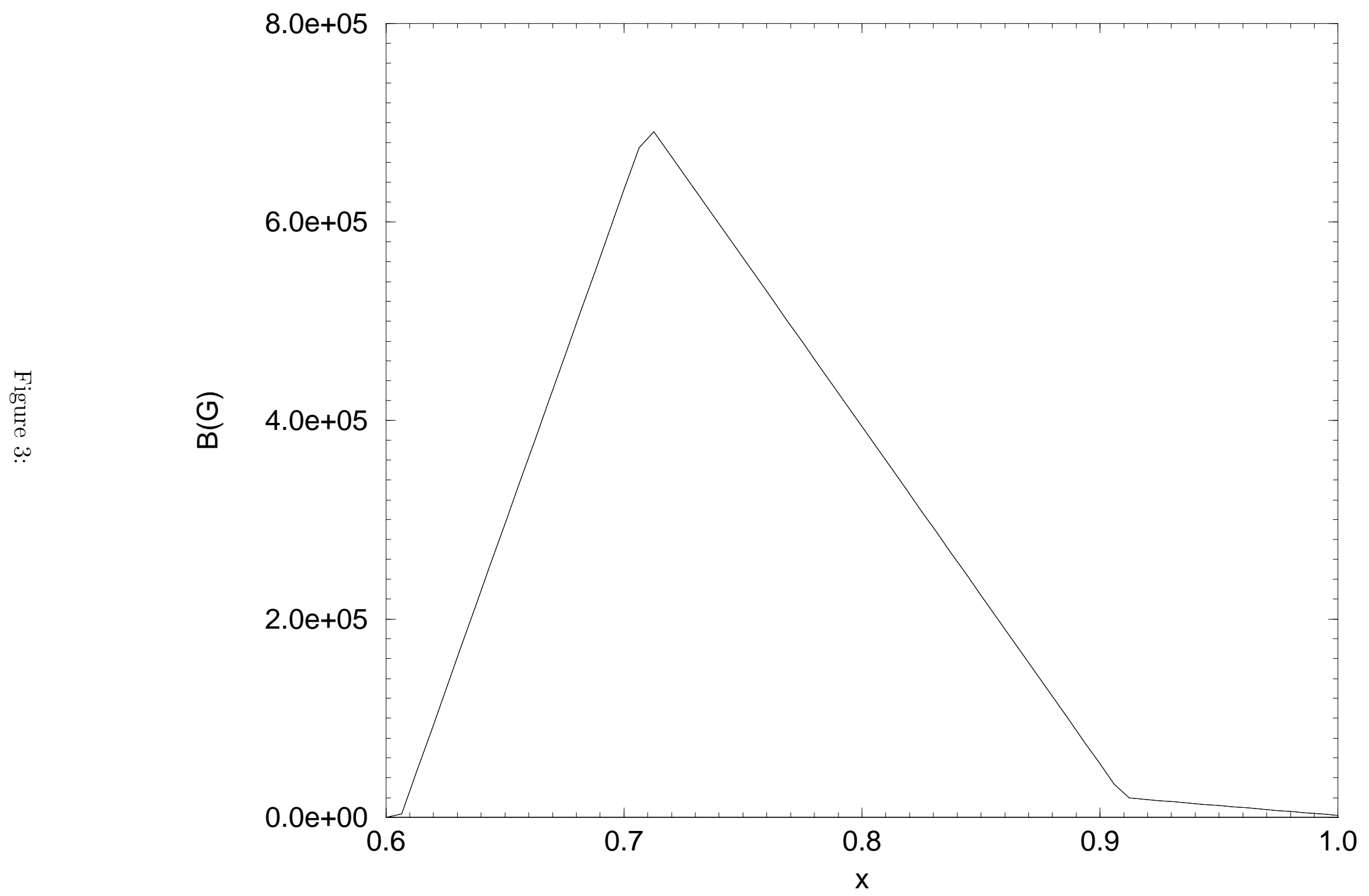




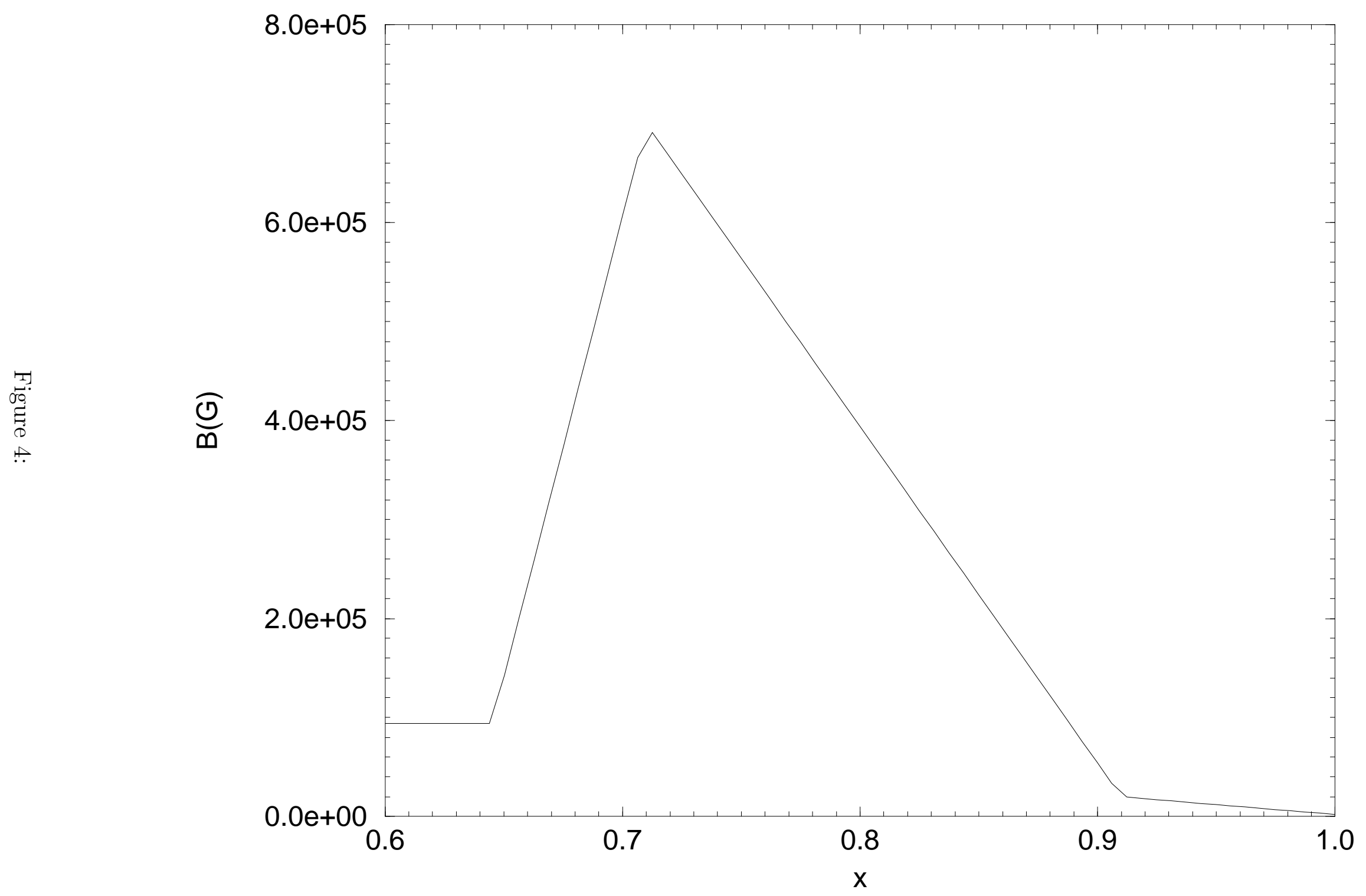




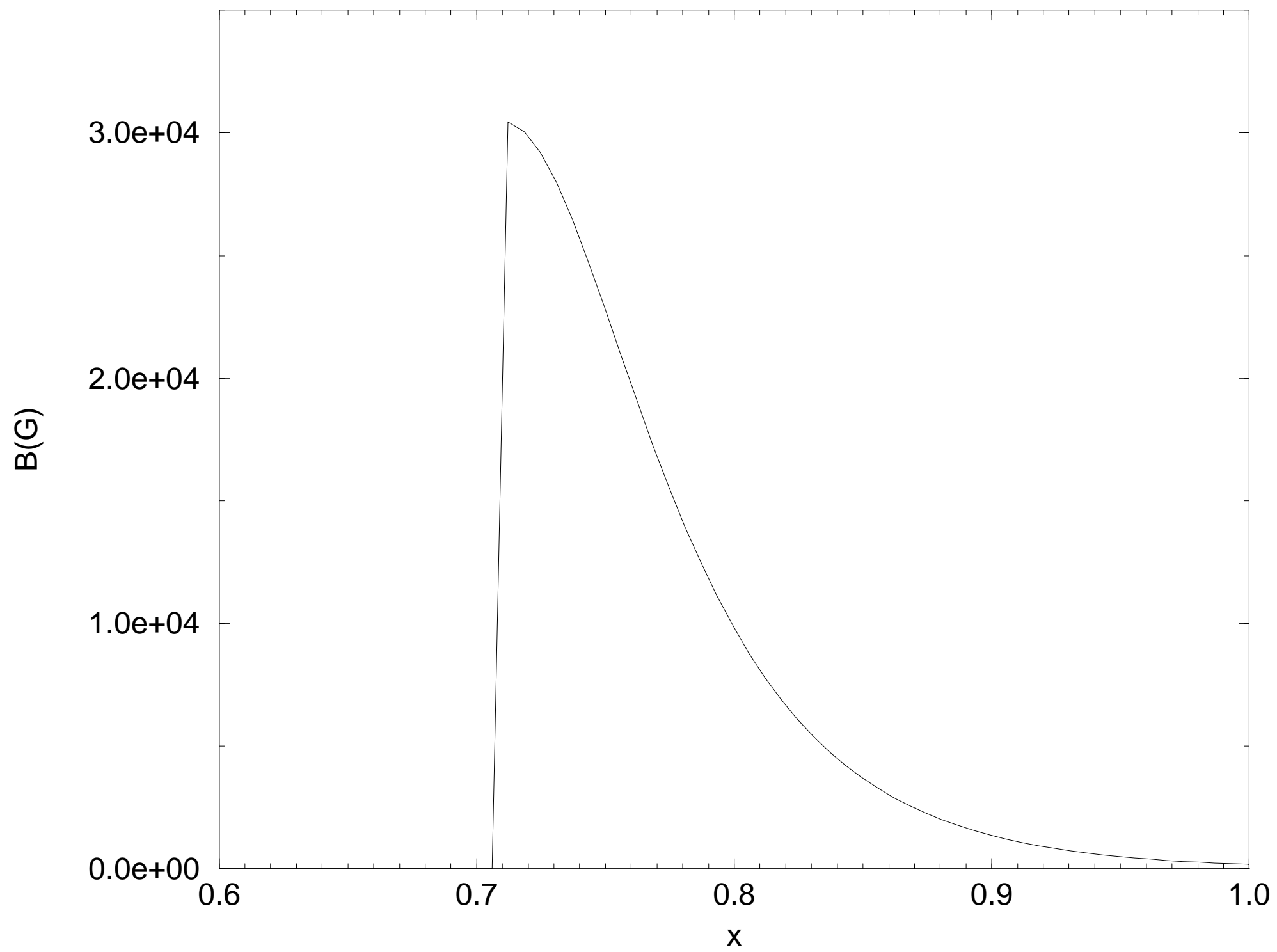




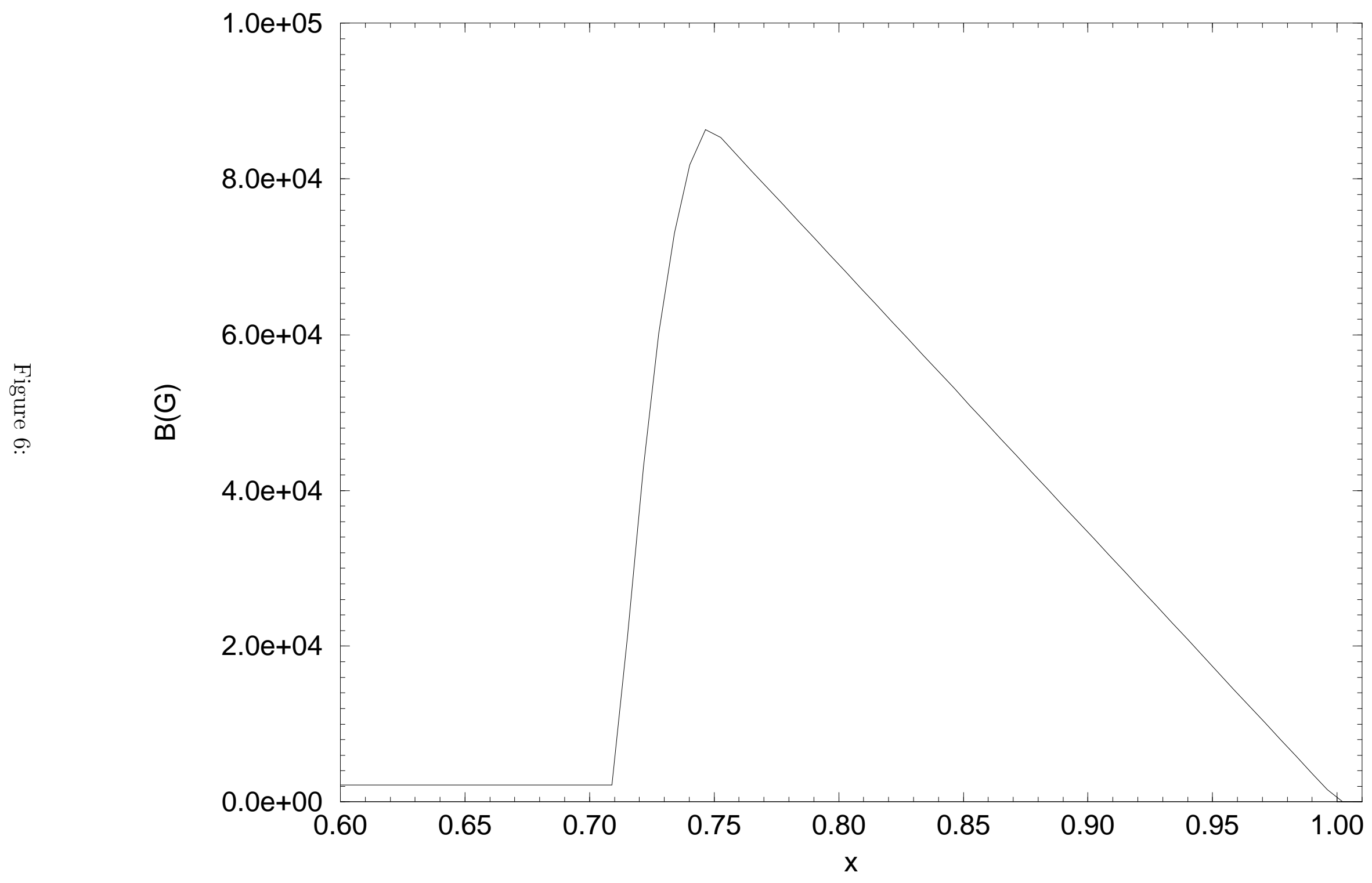




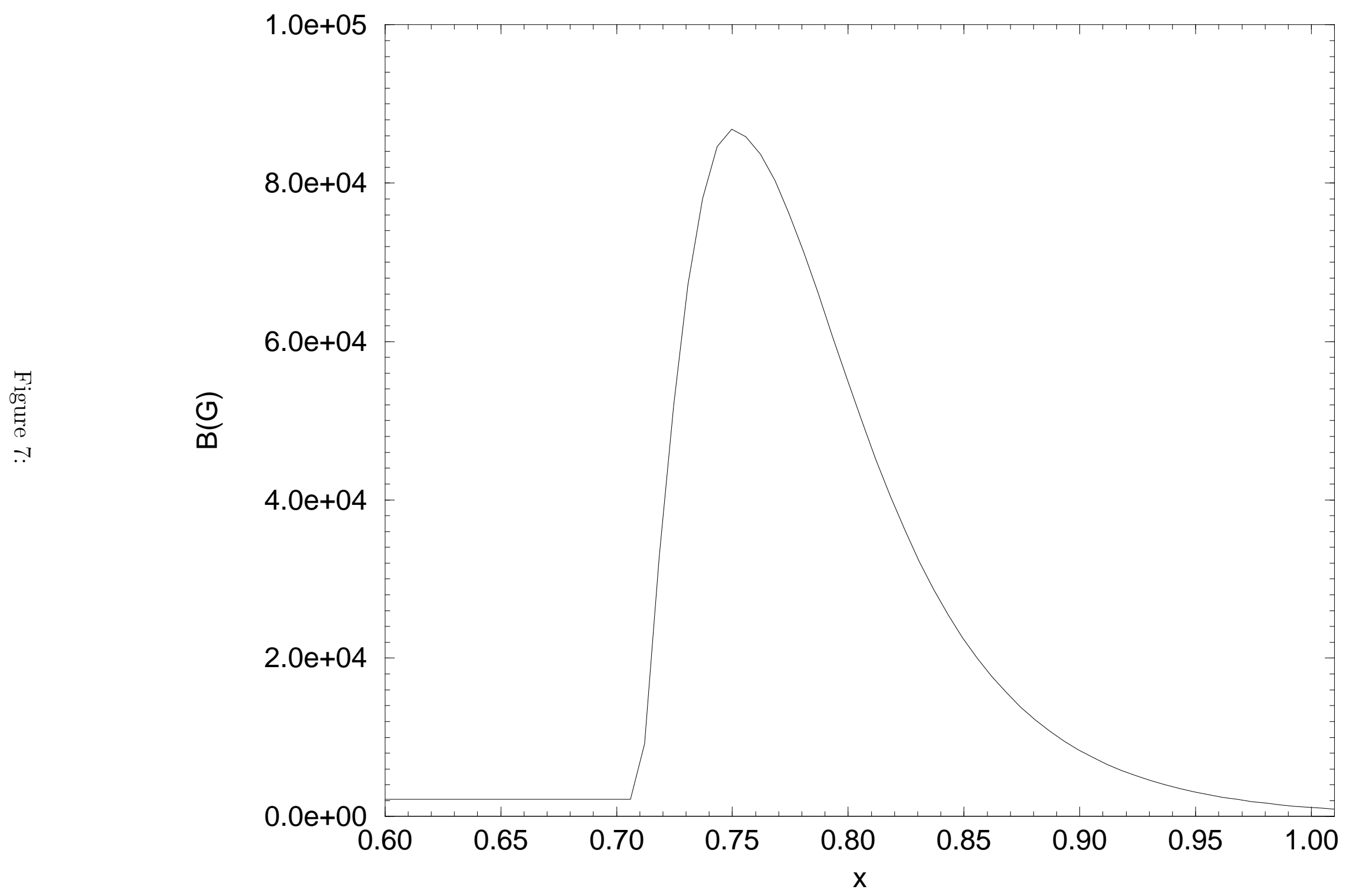




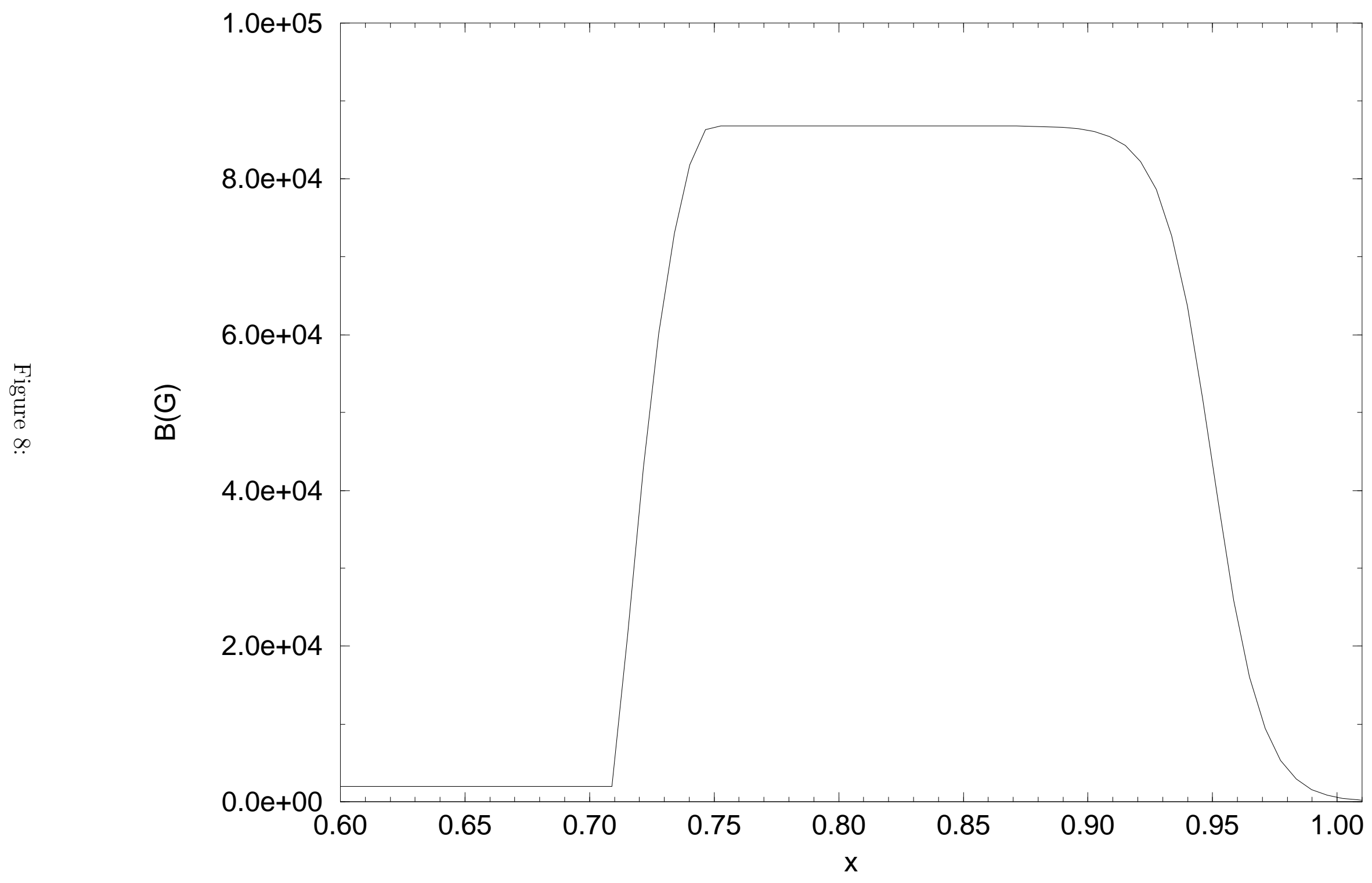

